\title{
Quantifying quantum discord and entanglement of formation via unified purifications
}

\author{
Li-Xiang Cen, ${ }^{1,2}$ Xin-Qi Li, ${ }^{3,1,2}$ Jiushu Shao,, ${ }^{4,2}$ and YiJing Yan ${ }^{2}$ \\ ${ }^{1}$ Department of Physics, Sichuan University, Chengdu 610065, China \\ ${ }^{2}$ Department of Chemistry, Hong Kong University of Science and Technology, Kowloon, Hong Kong \\ ${ }^{3}$ Department of Physics, Beijing Normal University, Beijing 100875, China \\ ${ }^{4}$ College of Chemistry, Beijing Normal University, Beijing 100875,China
}

(Received 19 July 2010; published 17 May 2011)

\begin{abstract}
We propose a scheme to evaluate the amount of quantum discord and entanglement of formation for mixed states and reveal their ordering relation via an intrinsic relationship between the two quantities distributed in the purification of different states. This approach enables us to achieve analytical expressions of the two measures for some quantum states, such as an arbitrary two-qubit density matrix reduced from pure three-qubit states and a class of rank- 2 mixed states of $4 \times 2$ systems. Moreover, we apply the scheme to characterize fully the dynamic behavior of quantum correlations for the specified physical systems under decoherence.
\end{abstract}

DOI: 10.1103/PhysRevA.83.054101

PACS number(s): 03.65.Ud, 03.65.Ta, 03.65.Yz

Quantum correlation is a fundamental resource for quantum information processing and it has been the subject of intensive studies in recent decades. The nonlocality aspect of quantum correlations, termed entanglement, was first singled out as the characteristic trait of quantum mechanics that is inaccessible to classical objects [1]. It is widely believed that entanglement constitutes the key ingredient leading to the power of quantum computation [2,3]. Operationally, entangled states are those that cannot be prepared through local operations and classical communication between two parties [4]. In other words, they cannot be written in a separable form: $\rho_{A B} \neq \sum_{k} p_{k} \rho_{A}^{k} \otimes \rho_{B}^{k}$. The amount of entanglement of $\rho_{A B}$ shared in the two parties is usually defined by the entanglement of formation (EOF) [5], i.e., the minimal average entanglement of pure state ensembles to create $\rho_{A B}: E\left(\rho_{A B}\right) \equiv \min \sum_{k} p_{k} E\left(\left|\psi_{k}\right\rangle\right)$, where the minimum is taken over all possible decompositions $\rho_{A B}=\sum_{k} p_{k}\left|\psi_{k}\right\rangle\left\langle\psi_{k}\right|$, and $E\left(\left|\psi_{k}\right\rangle\right)$ is the entropy entanglement of the pure state $\left|\psi_{k}\right\rangle$ in the ensemble.

A different notion of measure, quantum discord $Q_{A B}$, has also been proposed $[6,7]$ to characterize quantum correlations based on an information-theoretic measure of mutual information. Quantum discord is calculated through quantifying the classicality and/or nonclassicality in the total mutual information $I\left(\rho_{A B}\right) \equiv S\left(\rho_{A}\right)+S\left(\rho_{B}\right)-S\left(\rho_{A B}\right)$. It is defined as $Q_{A B} \equiv I\left(\rho_{A B}\right)-J_{A B}$, the discrepancy between $I\left(\rho_{A B}\right)$ and its classical counterpart $J_{A B} \equiv S\left(\rho_{B}\right)-S\left(\rho_{B} \mid A\right)$, where $S\left(\rho_{B} \mid A\right)$ is the conditional entropy, i.e., the minimal average entropy of $B$, given measurements on $A$ [cf. Eq. (1)]. For pure bipartite states $\left|\psi_{A B}\right\rangle$, the discord is equal to the entropy entanglement, $Q_{A B}=E\left(\left|\psi_{A B}\right\rangle\right) \equiv-\operatorname{tr}\left(\rho_{\mathrm{A}} \log _{2} \rho_{\mathrm{A}}\right)$, but for mixed states the discord is generally not identical to the EOF except for particular cases. The conceptual difference between these two measures has motivated extensive studies recently, e.g., on their roles in the performance of information processing $[8,9]$ and their relations to dynamics under decoherence [10]. On the other hand, the quantitative evaluation of quantum discord involves an optimization procedure similar to that in the EOF. The absence of a tractable method to tackle it makes comparison of these two measures and relevant studies very obscure. Until now, the explicit expression of quantum discord has only been obtained for certain classes of two-qubit states [11,12].
In this Brief Report, we propose a scheme to evaluate the amount of quantum discord and EOF via an intrinsic relation between the two quantities distributed in the purification of given mixed states. The same relation of duality had been revealed by Koashi and Winter in a context to explore the monogamous nature of entanglement measures [13]. Here we demonstrate that the elicited trilateral relationship can be applied to quantify the quantum discord and EOF and their ordering relation. In particular, the scheme enables us to fully characterize the discord and EOF for certain quantum states, e.g., an arbitrary two-qubit state reduced from pure three-qubit states and a class of rank- 2 mixed states of $4 \times 2$ systems. Additionally, we further apply the quantification scheme to describe the dynamic behavior of quantum correlations for specified systems under decoherence.

Conditional entropy versus entanglement of formation in purifications. Let us start with the conditional entropy

$$
S\left(\rho_{B} \mid A\right) \equiv \min _{\left\{A_{k}\right\}} \sum_{k} p_{k} S\left(\rho_{B}^{k} \mid A_{k}\right),
$$

the central ingredient contained in the definition of the quantum discord. We discriminate between two different operations of $\left\{A_{k}\right\}$, the von Neumann projective measurements $\left\{\Pi_{k} \equiv\right.$ $\left.\left|k_{A}\right\rangle\left\langle k_{A}\right| ; k=1, \ldots, d\right\}$ and the generalized positive operatorvalued measurements (POVMs) $\left\{\mathcal{A}_{k}\right\}$ satisfying $\sum_{k} \mathcal{A}_{k}^{\dagger} \mathcal{A}_{k}=$ $I$. Their corresponding definitions of conditional entropy are denoted by $S_{I}$ and $S_{I I}$, respectively. Accordingly, we have two kinds of definitions for quantum discord:

$$
Q_{A B}^{I, I I}=S\left(\rho_{A}\right)+S_{I, I I}\left(\rho_{B} \mid A\right)-S\left(\rho_{A B}\right) .
$$

To proceed we invoke the so-called purification $\left|\Psi_{A B C}\right\rangle$ of $\rho_{A B}$, whose partial trace on the ancillary $C$ gives rise to $\operatorname{tr}_{C}\left|\Psi_{A B C}\right\rangle\left\langle\Psi_{A B C}\right|=\rho_{A B}$. Suppose that $C$ has a dimension equal to the rank of $\rho_{A B}$, then all such purifications should be equivalent up to local unitary transformations on $C$. With the notion of $\left|\Psi_{A B C}\right\rangle$, each outcome of the orthogonal projective measurement $\left\{\left|k_{A}\right\rangle\left\langle k_{A}\right|\right\}$ will be associated with a relative state $\left|\psi_{B C}^{k}\right\rangle=\left\langle k_{A} \mid \Psi_{A B C}\right\rangle / \sqrt{p_{k}}$, where $p_{k}$ is the probability of the $k$ th outcome. The entropy of $B$ (as that of $C$ ), conditioned to the $k$ th outcome, is precisely captured by the entropy entanglement in $\left|\psi_{B C}^{k}\right\rangle: S\left(\rho_{B}^{k} \mid A_{k}\right)=E\left(\left|\psi_{B C}^{k}\right\rangle\right)=S\left(\rho_{C}^{k} \mid A_{k}\right)$. 
Furthermore, because the set of relative states $\left\{p_{k},\left|\psi_{B C}^{k}\right\rangle\right\}$ actually forms an ensemble that realizes $\rho_{B C}$, namely, $\rho_{B C}=$ $\sum_{k} p_{k}\left|\psi_{B C}^{k}\right\rangle\left\langle\psi_{B C}^{k}\right|$, we obtain

$$
S_{I}\left(\rho_{B} \mid A\right)=\min _{\left\{\Pi_{k}\right\}} \sum_{k} p_{k} E\left(\left|\psi_{B C}^{k}\right\rangle\right) \equiv E^{[d]}\left(\rho_{B C}\right),
$$

where $E^{[d]}\left(\rho_{B C}\right)$ defines a $d$-component EOF, i.e., the minimal average entanglement of $\rho_{B C}$ over ensemble decompositions with only $d$ components. In general, $E^{[d]}\left(\rho_{B C}\right) \geqslant E\left(\rho_{B C}\right)$ since the minimization in the definition of $E\left(\rho_{B C}\right)$ is taken over all ensemble decompositions realizing $\rho_{B C}$ but the $d$-component ensemble decompositions via the outcome of projective measurements $\left\{\Pi_{k}\right\}$ on $A$ are only a portion of them.

On the other hand, the outcome via the complete set of POVMs $\left\{\mathcal{A}_{k}\right\}$ offers a distinct way to realize $\rho_{B C}$ because $\sum_{k} \operatorname{tr}_{A}\left(\mathcal{A}_{k}\left|\Psi_{A B C}\right\rangle\left\langle\Psi_{A B C}\right| \mathcal{A}_{k}^{\dagger}\right)=\rho_{B C}$. Note that the ensemble generated here comprises also those mixed states. It follows from the concavity property of the von Neumann entropy that the minimum of the conditional entropy of Eq. (1) is always reached with ensembles of pure states [13]. Consequently, for the quantity $S_{I I}\left(\rho_{B} \mid A\right)$ in which POVMs on $A$ are assumed, there exists

$$
S_{I I}\left(\rho_{B} \mid A\right)=E\left(\rho_{B C}\right)=S_{I I}\left(\rho_{C} \mid A\right) .
$$

To make clear that a POVM realizing the optimal ensemble with minimal average entanglement $E\left(\rho_{B C}\right)$ can always be constructed, it is instructive to invoke the following fact: By including an external system $E$ with an arbitrary high dimension and performing joint unitary evolutions on $A$ and $E$, all ensembles of pure states reproducing $\rho_{B C}$ can be generated via the outcome $\left\{p_{k^{\prime} k},\left|\psi_{B C}^{k^{\prime} k}\right\rangle\right\}$ associated with von Neumann measurements $\left\{\left|k_{A}^{\prime} k_{E}\right\rangle\left\langle k_{A}^{\prime} k_{E}\right|\right\}$ on $A$ and $E$, where

$$
\left|\psi_{B C}^{k^{\prime} k}\right\rangle=\left\langlek _ { A } ^ { \prime } k _ { E } \left| U_{A E}\left(\left|\Psi_{A B C}\right\rangle \otimes\left|0_{E}\right\rangle\right) / \sqrt{p_{k^{\prime} k}} .\right.\right.
$$

The action of a general POVM $\left\{\mathcal{A}_{k}\right\}$ on $\left|\Psi_{A B C}\right\rangle$ could be described in a similar way as

$$
\begin{aligned}
\mathcal{A}_{k}\left|\Psi_{A B C}\right\rangle & =\left\langlek _ { E } \left| U_{A E}\left(\left|\Psi_{A B C}\right\rangle \otimes\left|0_{E}\right\rangle\right)\right.\right. \\
& =\sum_{k^{\prime}=1}^{d_{A}} \sqrt{p_{k^{\prime} k}}\left|k_{A}^{\prime}\right\rangle\left|\psi_{B C}^{k^{\prime} k}\right\rangle .
\end{aligned}
$$

To output the ensemble of pure states of Eq. (5) through a POVM action, we revise the POVM (6) by partitioning the operators $\left\{\mathcal{A}_{k}\right\}$ into $\left\{\mathcal{A}_{k^{\prime}, k}=\left|k_{A}^{\prime}\right\rangle\left\langle k_{A}^{\prime}\right| \mathcal{A}_{k} ; k^{\prime}=1, \ldots, d\right\}$ with $d$ the dimension of $A$. It is then readily seen that the new POVMs $\left\{\mathcal{A}_{k^{\prime}, k}\right\}$ could give rise to the general ensemble of relative states in Eq. (5): $\mathcal{A}_{k^{\prime}, k}\left|\Psi_{A B C}\right\rangle \rightarrow\left\{p_{k^{\prime} k},\left|\psi_{B C}^{k^{\prime} k}\right\rangle\right\}$.

The relationship between (3) and (4) suggests a scheme to quantify the quantum discord and EOF (or the $d$-component EOF) through building purifications of given mixed states. For example, because the EOF of mixed states of two-qubit systems has already been perfectly resolved [14], it indicates that the discord of all $n \times 2(n \geqslant 2)$ density matrices with no more than two nonzero eigenvalues can be achieved accordingly. Furthermore, by employing Eq. (4) and the similar relation for $S_{I I}\left(\rho_{A} \mid C\right)$, one obtains

$$
Q_{A B}^{I I}-E\left(\rho_{A B}\right)=Q_{A C}^{I I}-Q_{C A}^{I I} .
$$

At this stage, it is clear that the ordering relation of the two quantities, $Q_{A B}^{I I}$ and $E\left(\rho_{A B}\right)$, is essentially connected to the asymmetric property of the quantum discord, reflected via the lateral $\rho_{A C}$ (or $\rho_{B C}$ if we consider $Q_{B A}^{I I}$ instead) in the purification. In what follows we shall apply the scheme on some concrete quantum states so as to evaluate the amount of their discord and EOF and the ordering relation.

Quantum discord and entanglement in pure states of three qubits. It is correct to apply the above scheme to solve the issue for arbitrary two-qubit density matrices reduced from three-qubit pure states. Because a three-qubit pure state can be expressed generally as [15]

$$
\begin{aligned}
\left|\psi_{A B C}\right\rangle= & \lambda_{0}|000\rangle+\lambda_{1} e^{i \varphi}|010\rangle+\lambda_{2}|011\rangle \\
& +\lambda_{3}|110\rangle+\lambda_{4}|111\rangle,
\end{aligned}
$$

the EOF of any pair could be worked out explicitly via the formula developed by Wootters [14]:

$$
E\left(\rho_{X Y}\right)=-x \log _{2} x-(1-x) \log _{2}(1-x),
$$

where $x=\frac{1}{2}\left[1+\sqrt{1-C^{2}\left(\rho_{X Y}\right)}\right]$ and the concurrence is obtained as

$$
\begin{gathered}
C\left(\rho_{A B}\right)=2 \lambda_{0} \lambda_{3}, \quad C\left(\rho_{B C}\right)=2 \lambda_{0} \lambda_{2}, \\
C\left(\rho_{A C}\right)=2\left|\lambda_{2} \lambda_{3}-\lambda_{1} \lambda_{4} e^{i \varphi}\right| .
\end{gathered}
$$

Since the optimal ensemble decomposition realizing the minimal average entanglement for each $\rho_{X Y}$ here has only two components, it can be achieved by projective measurements on the third party $Z$. So, $S_{I}\left(\rho_{X} \mid Z\right)=S_{I I}\left(\rho_{X} \mid Z\right)=E\left(\rho_{X Y}\right)$. As the conditional entropy has been derived, the discord of each $\rho_{Z X}$ can be directly obtained according to Eq. (2).

To illustrate the relation (7), we note that for the case $\lambda_{2}=$ $\lambda_{3}$, the state $\rho_{A C}$ is symmetric under permutation and hence $Q_{A C}=Q_{C A}$. Consequently,

$$
Q_{A B}=E\left(\rho_{A B}\right)=-\sum_{+,-} \frac{1 \pm \Delta}{2} \log _{2} \frac{1 \pm \Delta}{2},
$$

where $\Delta=\sqrt{1-\lambda_{0}^{2} \lambda_{2}^{2}}$.

Quantum discord in $4 \times 2$ systems with no more than two nonzero eigenvalues. The discord of any $n \times 2$ system $\rho_{A B}$ with only two nonzero eigenvalues can be recast to that of a $4 \times$ 2 system. It can be readily seen that for the purification $\left|\psi_{A B C}\right\rangle$ of $\rho_{A B}$ the relative system $C$ could have two dimensions, so that the reduced density matrix $\rho_{B C}$ (and hence $\rho_{A}$ ) has at most four nonzero eigenvalues. Since the EOF of two binary systems $\rho_{B C}$ has been perfectly resolved, it leads to the fact that the discord of any such states can be explicitly obtained. To illustrate, we present below an example of a four-parameter family of rank-2 states formed as

$$
\rho_{A B}=p_{1}\left|\psi_{1}\right\rangle\left\langle\psi_{1}\left|+p_{2}\right| \psi_{2}\right\rangle\left\langle\psi_{2}\right|,
$$

where

$$
\begin{gathered}
\left|\psi_{1}\right\rangle=\cos \phi|00\rangle+\sin \phi|11\rangle, \\
\left|\psi_{2}\right\rangle=\sin \phi\left|a_{3} 0\right\rangle+\cos \phi\left|a_{4} 1\right\rangle
\end{gathered}
$$

are two normalized eigenvectors of $\rho_{A B}$ with

$$
\begin{aligned}
& \left|a_{3}\right\rangle=\cos \theta_{1}|1\rangle+\sin \theta_{1}|2\rangle, \\
& \left|a_{4}\right\rangle=\cos \theta_{2}|0\rangle+\sin \theta_{2}|3\rangle .
\end{aligned}
$$


To calculate the quantum discord in Eq. (2), it is easy to obtain $S\left(\rho_{A B}\right)=-\sum_{i=1}^{2} p_{i} \log _{2} p_{i}$ and $S\left(\rho_{A}\right)=-\sum_{i=1}^{4} \lambda_{i} \log _{2} \lambda_{i}$, where

$$
\begin{aligned}
& \lambda_{1,2}=\frac{1}{2} \sin ^{2} \phi\left(1 \pm \sqrt{1-4 p_{1} p_{2} \sin \theta_{1}^{2}}\right), \\
& \lambda_{3,4}=\frac{1}{2} \cos ^{2} \phi\left(1 \pm \sqrt{1-4 p_{1} p_{2} \sin \theta_{2}^{2}}\right) .
\end{aligned}
$$

The conditional entropy $S\left(\rho_{B} \mid A\right)$ can be derived via the equality of Eq. (4) and the purification herein could be expressed as $\left|\psi_{A B C}\right\rangle=\sqrt{p_{1}}\left|\psi_{1}\right\rangle\left|0_{C}\right\rangle+\sqrt{p_{1}}\left|\psi_{2}\right\rangle\left|1_{C}\right\rangle$. Note that the optimal ensemble decomposition realizing $E\left(\rho_{B C}\right)$ has four components [14]; this very ensemble can be achieved through outcomes of the projective measurements on $A$ because $\operatorname{dim} A=4$. This leads to the relation $S_{I}\left(\rho_{B} \mid A\right)=$ $S_{I I}\left(\rho_{B} \mid A\right)=E\left(\rho_{B C}\right)$ for the present system. Explicitly, its value is given by the formula of Eq. (9) and the corresponding concurrence is now obtained as $C\left(\rho_{B C}\right)=\max \left\{0,2 \lambda_{m}^{c}-\right.$ $\left.\sum_{i=1}^{4} \lambda_{i}^{c}\right\}$, where $\lambda_{m}^{c}$ is the largest of

$$
\begin{aligned}
& \lambda_{1,2}^{c}=\frac{1}{2} \sin ^{2} \phi\left[\sqrt{1-\left(p_{1}-p_{2}\right)^{2}} \pm 2 \sqrt{p_{1} p_{2}} \cos \theta_{1}\right], \\
& \lambda_{3,4}^{c}=\frac{1}{2} \cos ^{2} \phi\left[\sqrt{1-\left(p_{1}-p_{2}\right)^{2}} \pm 2 \sqrt{p_{1} p_{2}} \cos \theta_{2}\right] .
\end{aligned}
$$

Deriving entanglement of formation via quantum discord. The quantitative calculation of the EOF for mixed states is notoriously difficult and the explicit expression is derived only for two-qubit systems [14] and very limited cases of high-dimensional systems [16]. Notably, the derivation of the relation of quantum correlations in tripartite purifications suggests also a distinct way to calculate the EOF by virtue of the conditional entropy. In particular, let us consider the states $\rho_{A B}$ of a $4 \times 2$ system given by Eq. (12). By examining the purification $\left|\psi_{A B C}\right\rangle$ it is seen that the resulting state $\rho_{B C}$ is an $X$-class two-qubit state, with the nonzero elements being

$$
\begin{gathered}
\rho_{B C}^{11}=p_{1} \cos ^{2} \phi, \quad \rho_{B C}^{14}=\rho_{B C}^{41}=\sqrt{p_{1} p_{2}} \cos ^{2} \phi \cos \theta_{2}, \\
\rho_{B C}^{22}=p_{2} \sin ^{2} \phi, \quad \rho_{B C}^{23}=\rho_{B C}^{32}=\sqrt{p_{1} p_{2}} \sin ^{2} \phi \cos \theta_{1}, \\
\rho_{B C}^{33}=p_{1} \sin ^{2} \phi, \quad \rho_{B C}^{44}=p_{2} \cos ^{2} \phi .
\end{gathered}
$$

In the two-qubit case, it has been proved [17] that the projective measurement is the optimal POVM to minimize the conditional entropy, and its evaluation for the $X$-class state has been resolved in Ref. [12]. According to the scheme proposed previously, this means $E\left(\rho_{A B}\right)=E^{[2]}\left(\rho_{A B}\right)$ and its expression can be explicitly calculated. In particular, for the case $p_{1}=p_{2}=1 / 2$, the state $\rho_{B C}$ reduces to a Bell-diagonal state (i.e., $\left.\rho_{B}=\rho_{C}=I_{2} / 2\right)$. The conditional entropy $S\left(\rho_{B} \mid C\right)$ in this case has a concise expression [11] and so does the EOF of $\rho_{A B}$ :

$$
E\left(\rho_{A B}\right)=S\left(\rho_{B} \mid C\right)=-\sum_{+,-} \frac{1 \pm \chi}{2} \log _{2} \frac{1 \pm \chi}{2},
$$

where $\chi=\max \left\{\left|\chi_{1}\right|,\left|\chi_{2}\right|,\left|\chi_{3}\right|\right\}$, with

$$
\chi_{1}=-\cos 2 \phi, \quad \chi_{2,3}=\cos ^{2} \phi \cos \theta_{2} \pm \sin ^{2} \phi \cos \theta_{1} .
$$

The calculation above actually offers a full characterization of the state (12) for both its quantum discord and EOF.

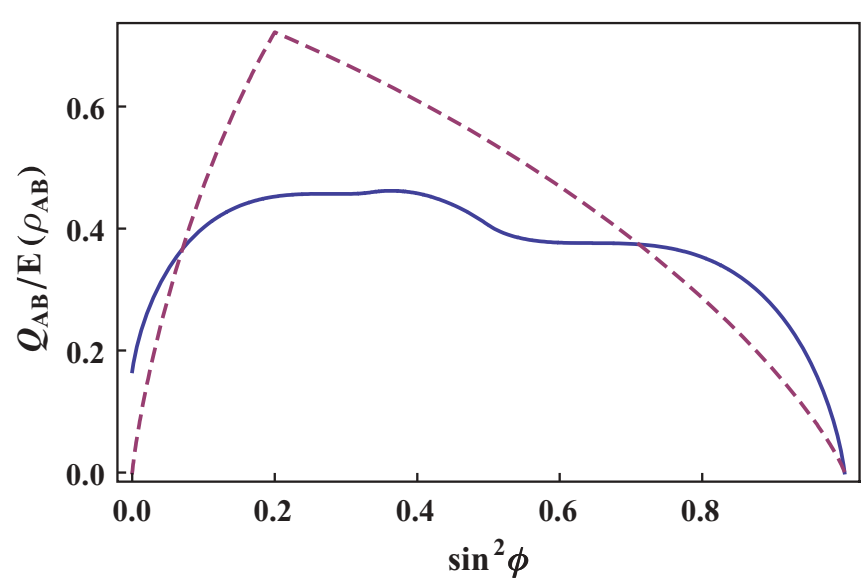

FIG. 1. (Color online) Quantum discord (solid line) vs EOF (dashed line) in the $4 \times 2$ system specified by Eq. (12), where the coefficients are given by $p_{1,2}=1 / 2, \theta_{1}=0$, and $\theta_{2}=\pi / 3$. Both quantities are shown to be discrete functions of $k_{1}^{2}$, and the partition of the range is depicted as $\sin ^{2} \phi \in(0,1 / 3),(1 / 3,1 / 2),(1 / 2,1)$ for the discord, and $\sin ^{2} \phi \in(0,1 / 5),(1 / 5,1)$ for the EOF, respectively.

We plot in Fig. 1 the two quantities as functions of $\phi$, where $p_{1,2}=1 / 2, \theta_{1}=0$, and $\theta_{2}=\pi / 3$. The result shows that the EOF is lower than the discord as $\sin ^{2} \phi \lesssim 0.070$ and $\sin ^{2} \phi \gtrsim 0.711$ and larger than the discord in the range $0.070<\sin ^{2} \phi<0.711$.

As the final proposal of this Brief Report, we apply the derived results of quantification on the discord and EOF to characterize their dynamic behavior in a typical physical process. Consider a two-qubit system initially prepared in the state $\left|\psi_{A B}\right\rangle=\alpha|00\rangle+\beta|11\rangle$, with $|\alpha|^{2}+|\beta|^{2}=1$. Suppose that one of the qubits is subjected to a phase-damping environment (which can be realized, e.g., in optical systems as one of the photons passes through a phase-damping channel [18]). The system will then evolve as

$$
\begin{aligned}
\rho_{A B}= & |\alpha|^{2}|00\rangle\left\langle\left. 00|+| \beta\right|^{2} \mid 11\right\rangle\langle 11| \\
& +e^{-\gamma t}\left(\alpha \beta^{*}|00\rangle\left\langle 11\left|+\alpha^{*} \beta\right| 11\right\rangle\langle 00|\right),
\end{aligned}
$$

with the phase-damping rate $\gamma$. Since the state $\rho_{A B}$ has only two nonzero eigenvalues, its quantum discord can be worked out explicitly via the above derived results with the purification $\left|\psi_{A B C}\right\rangle$ of the three-qubit system. It turns out that $\rho_{B C}$ and $\rho_{A C}$ reduced from $\left|\psi_{A B C}\right\rangle$ are separable states. Therefore the conditional entropy $S_{I, I I}\left(\rho_{B} \mid A\right)=E\left(\rho_{B C}\right)=0$. The quantum discord of $\rho_{A B}$ is obtained simply as $Q_{A B}=S\left(\rho_{A}\right)-S\left(\rho_{A B}\right)$, which is fully specified by the spectra of $\rho_{A}$ and $\rho_{A B}$, expressed in detail as $\left\{|\alpha|^{2},|\beta|^{2}\right\}$ and $\frac{1}{2} \pm \sqrt{\frac{1}{4}-|\alpha \beta|^{2}\left(1-e^{-2 \gamma t}\right)}$, respectively. We plot in Fig. 2 the dynamic behavior of the quantum discord $Q_{A B}$ and the difference between it and the EOF of $\rho_{A B}$, as a function of $|\alpha|^{2}$ and $p=1-e^{-\gamma t}$. The EOF is obtained from Eq. (9), in which the concurrence of the present system is $C\left(\rho_{A B}\right)=2|\alpha \beta| e^{-\gamma t}$. It is shown that the EOF is always larger than the discord in the specified phase-damping process of this model. 

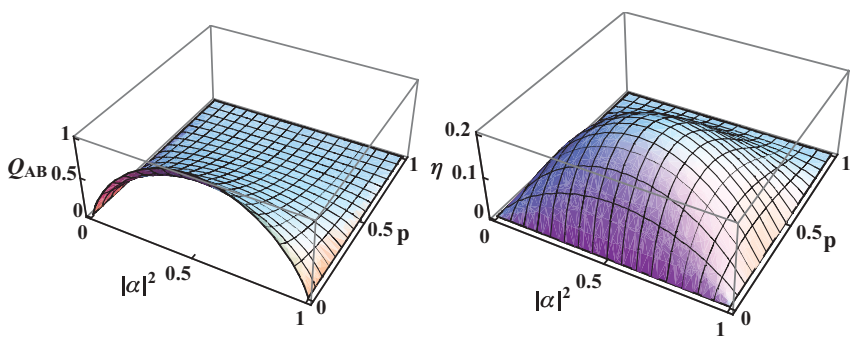

FIG. 2. (Color online) Dynamic behavior of quantum correlations under a phase-damping condition [cf. Eq. (20)]. Plotted are the quantum discord $Q_{A B}$ (left) and the difference between it and the EOF, $\eta \equiv E\left(\rho_{A B}\right)-Q_{A B}$ (right), as functions of $|\alpha|^{2}$ and $p=1-e^{-\gamma t}$.

In summary, two measures of quantum correlations, quantum discord and EOF, have been investigated via the unified purification of given mixed quantum states. We show that their amount and ordering relation can be evaluated via the relationship of their distribution in different partners of the purification. The scheme is then exploited to achieve analytical expressions of the quantum discord and the EOF for some quantum states, including arbitrary two-qubit density matrices reduced from pure three-qubit states and a class of rank-2 mixed states of $4 \times 2$ systems. In its application to physical systems, we show that the derived result of quantification on the discord and EOF enables us to characterize their dynamic behavior in certain typical physical processes. Finally, we mention other applications proposed recently concerning the duality relation of the quantum discord and EOF, e.g., the resolution of the EOF for a class of Gaussian states [19], and their role in relation to the power of deterministic quantum computation [20].

Support from the National Natural Science Foundation of China (Grants No. 10604043 and No. 10874254) and RGC Hong Kong (Grant No. 604709) is acknowledged.
[1] E. Schrödinger, Proc. Cambridge Philos. Soc. 31, 555 (1935).

[2] P. W. Shor, SIAM J. Comput. 26, 1484 (1997).

[3] L. K. Grover, Phys. Rev. Lett. 79, 325 (1997).

[4] R. F. Werner, Phys. Rev. A 40, 4277 (1989).

[5] C. H. Bennett, G. Brassard, S. Popescu, B. Schumacher, J. A. Smolin, and W. K. Wootters, Phys. Rev. Lett. 76, 722 (1996).

[6] L. Henderson and V. Vedral, J. Phys. A 34, 6899 (2001).

[7] H. Ollivier and W. H. Zurek, Phys. Rev. Lett. 88, 017901 (2001); W. H. Zurek, Phys. Rev. A 67, 012320 (2003).

[8] E. Knill and R. Laflamme, Phys. Rev. Lett. 81, 5672 (1998); A. Datta, A. Shaji, and C. M. Caves, ibid. 100, 050502 (2008); B. P. Lanyon, M. Barbieri, M. P. Almeida, and A. G. White, ibid. 101, 200501 (2008); A. Datta and S. Gharibian, Phys. Rev. A 79, 042325 (2009).

[9] M. Piani, P. Horodecki, and R. Horodecki, Phys. Rev. Lett. 100, 090502 (2008); M. Piani, M. Christandl, C. E. Mora, and P. Horodecki, ibid. 102, 250503 (2009).

[10] J. Maziero, L. C. Celeri, R. M. Serra, and V. Vedral, Phys. Rev. A 80, 044102 (2009); T. Werlang, S. Souza, F. F. Fanchini, and C. J. Villas Boas, ibid. 80, 024103 (2009); F. F. Fanchini,
T. Werlang, C. A. Brasil, L. G. E. Arruda, and A. O. Caldeira, ibid. 81, 052107 (2010); B. Wang, Z.-Y. Xu, Z.-Q. Chen, and M. Feng, ibid. 81, 014101 (2010).

[11] S. Luo, Phys. Rev. A 77, 042303 (2008).

[12] M. Ali, A. R. P. Rau, and G. Alber, Phys. Rev. A 81, 042105 (2010).

[13] M. Koashi and A. Winter, Phys. Rev. A 69, 022309 (2004).

[14] W. K. Wootters, Phys. Rev. Lett. 80, 2245 (1998).

[15] A. Acín, A. Andrianov, L. Costa, E. Jané, J. I. Latorre, and R. Tarrach, Phys. Rev. Lett. 85, 1560 (2000).

[16] B. M. Terhal and K. G. H. Vollbrecht, Phys. Rev. Lett. 85, 2625 (2000); K. G. H. Vollbrecht and R. F. Werner, Phys. Rev. A 64, 062307 (2001).

[17] S. Hamieh, R. Kobes, and H. Zaraket, Phys. Rev. A 70, 052325 (2004).

[18] J.-S. Xu et al., Nat. Commun. 1, 7 (2010).

[19] G. Adesso and A. Datta, Phys. Rev. Lett. 105, 030501 (2010); for Gaussian quantum discord, see also P. Giorda and Matteo G. A. Paris, ibid. 105, 020503 (2010).

[20] F. F. Fanchini, M. F. Cornelio, M. C. de Oliveira, and A. O. Caldeira, e-print arXiv:1006.2460. 